ISSN 1518-3483

Licenciado sob uma Licença Creative Commons

\title{
Reflexiones sobre la enseñanza de
} la política educativa y la formación de investigadores en el campo ${ }^{1}$

\author{
Reflexões sobre o ensino de política educativa e a \\ formação de pesquisadores
}

Reflections on the teaching of educational policy and researcher's formation

\author{
César Tello*
}

Universidad Nacional de Tres de Febrero (UNTREF), Buenos Aires, Argentina

\section{Resumen}

En este trabajo se presentan algunas reflexiones sobre la enseñanza de la política educacional y la formación de investigadores del campo. Se analiza el rol de los profesores del espacio curricular de la política educacional y el de los formadores de investigadores. Se plantea que los procesos formativos tanto en el grado como en el posgrado no puede

1 Esta es una nueva versión del artículo publicado originalmente en Revista Educação Pública, Cuiabá, v. 24, n. 55, p. 125-151, jan./abr. 2015

* CT: Doctor en Ciencias de la Educación. Director de la Red Latinoamericana de Estudios Epistemológicos en Política Educativa, e-mail: ctello@relepe.org 
estar desprendido del conocimiento de las matrices históricas del campo y sus epistemes de época. Argumentando que no existe un solo posicionamiento que deba ser transmitido en los procesos formativos a los jóvenes que estudian política educacional. Sino que es un desafio y un compromiso epistemológico para profesores y formadores mostrar, desarrollar y explicar los múltiples modos de abordar la política educacionales desde diversas matrices históricas, teóricas y epistemológicas.

Palabras clave: Enseñanza. Políticas educacionales. Modelos teóricos.

\section{Resumo}

Neste artigo algumas reflexões sobre o ensino de política educacional e formação de pesquisadores no campo são apresentadas. Investigam-se os papeis dos professores do espaço curricular da política educacional e dos formadores de investigadores. Afirma-se que os processos de formação, tanto de graduação e pós-graduação não podem ser separados do conhecimento das matrizes históricas do período do campo e de seus aspectos epistemológicos. Argumentando que não há uma posição única que deva ser transmitida no processo formativo de jovens que estudam a política de educação. Mas é um desafio e um compromisso epistemológico, para professores e formadores, desenvolver e explicar as muitas maneiras de abordar a política educacional a partir de várias matrizes históricas, teóricas e epistemológicas.

Palavras-chaves: Ensino. Políticas educativas. Modelos teóricos.

\section{Abstract}

In this paper some reflections on the teaching of educational policy and training of researchers in the field are presented. The teacher's role of curricular space of educational policy and researchers' trainers are analyzed. It is stated that the training processes in both degree and postgraduate studies cannot be detached from the knowledge of the historical matrices of the field and epistemic period. It is argued that there is no single position that is 
to be transmitted in the formative process to young people studying education policy. But it is a challenge and an epistemological for teachers and trainers, to develop and explain the many ways to address the educational policy from various historical, theoretical and epistemological matrices.

Keyword: Teaching. Educational policies. Theoretical models.

\section{Introducción}

En este artículo presentamos algunas consideraciones que intentan contribuir a la reflexión analítica de la enseñanza de la política educacional y la formación de investigadores del campo.

Debemos aclarar que no se trata de un texto con contribuciones del campo de la teoría y el desarrollo curricular de la política educacional - aunque los aportes curriculares siguen siendo necesarios para el campo - sino de un artículo que analiza, desde la mirada y posición de un investigador en política educacional la situación de la enseñanza en términos metodológicos y epistemológicos.

De modo analítico esgrimimos que las epistemes de época fueron caracterizando el campo y simultáneamente los modos de enseñar política educacional y formar investigadores. Esto es: el fuerte impulso de la perspectiva jurídico-institucionalista, los enfoques de la planificación en los orígenes del campo en Latinoamérica y las perspectivas unicausales se entrelazan y permanecen en los procesos curriculares y en la formación de los jóvenes investigadores. Estas epistemes de época confluyen en matrices históricas de un alto nivel descriptivo que se convierte en descripcionismo (TELLO, 2014).

Por lo tanto en este artículo argumentamos que las matrices históricas y las epistemes de época se presentan como modos, que se instalan en la formación y que terminan volviéndose las únicas miradas en términos de perspectivas teóricas y epistemológicas. 
Además, debemos considerar que el campo de la política educacional desde sus inicios surge como un campo eminentemente profesional, y así, el profesional en política educacional debía intervenir de un modo constante sobre la realidad. Esto llevó a priorizar el escenario de intervención del profesional en política educacional en detrimento de la profundización teórica, analítica y epistemológica de los instrumentos para pensar la realidad.

A sesenta años de la creación del campo en Latinoamérica ni los profesionales en política educacional ni los investigadores del campo han podido dar respuesta a la complejas problemáticas socio-políticoeducativas que se viven en la región. Quizá porque el campo profesional de la política educacional se encuentra demasiado alejado del estudio teórico para comprender la realidad, y, por otro lado, los investigadores se encuentran en un estado de dogmatismo teórico que solo permite describir los escenarios de la política educacional de la realidad latinoamericana sin llegar a comprenderla (TELLO; MAINARDES, 2015a).

En este marco, junto a otros investigadores de la región, hemos creado la Red Latinoamericana de Estudios Epistemológicos en política educativa, $\mathrm{ReLePe} \mathrm{e}^{2}$, para tratar de profundizar sobre estas cuestiones, que en ocasiones son dejadas a un lado por la urgencia de la realidad. Realidad que no debemos dejar de atender, pero mientras atendemos la realidad es un compromiso ético y político comprenderla. Diríamos: entender la realidad para atender la realidad.

Así presentamos en este artículo, en primer término, algunas consideraciones del campo de la política educacional como un campo que pertenece principalmente a las ciencias políticas y sociales y que de modo reticular convergen en ella la pedagogía, la sociología de la educación, la administración de la educación, en algunos casos toma componentes de la antropología de la educación y la economía de la educación. Luego reflexionamos sobre la matriz histórica de la institucionalización del campo de la política educacional y las epistemes de época y la crisis

$2<$ www.relepe.org $>$ 
epistémica de la política educativa que se manifiesta en los modos de enseñanza y formación de investigadores.

Así ingresamos al eje central del texto en cuanto a la enseñanza y la formación en política educacional para luego continuar con la compleja relación que, en su definición anglosajona, tienen los términos de polity, politics y policy, y que consideramos que de algún modo, en sus propias nominaciones, establecen un campo teórico congestionado y confuso, contribuyendo a la debilidad teórica del campo en Latinoamérica. De este modo, en nuestro planteo final, proponemos un pasaje de la enseñanza de las metodologías a las epistemetodologías de la política educacional (TELLO, 2012; TELLO; MAINARDES, 2015b), como un modo de abandonar los componentes técnicos que en general se presenta como instrumentos neutrales de la investigación en política educacional.

\section{El campo de la política educacional: entre las ciencias políticas y sociales}

Es necesario comprender que las ciencias sociales - o el pensamiento en ciencias sociales y ciencias políticas - se podría ubicar en Latinoamérica con el surgimiento de los Estados/Nación en la región. ${ }^{3}$ Por su parte las ciencias políticas deben entenderse en el marco del desarrollo de las ciencias sociales; el nacimiento de la ciencia política moderna se produce a través de la separación de los estudios políticos respecto de la matriz tradicional del derecho. Es en el año 1948 que se marca el hito fundacional del campo en la ciencia política: a pedido de la UNESCO (1949), se reúnen en París expertos y estudiosos con la finalidad

3 En este sentido Santiago Castro-Gómez (2000, p. 149) explica que el surgimiento de las ciencias sociales no es un fenómeno posterior a los modos de organización política de los surgentes Estados-Nación en Latinoamérica "[...] sino constitutivo de los mismos". Se requería de "[...] lineamientos científicos [...]" sobre la realidad para fundamentar los modos de gobernar. Es decir, "Sin el concurso de las ciencias sociales, el Estado moderno no se hallaría en la capacidad de ejercer control sobre la vida de las personas, definir metas colectivas a largo y a corto plazo, ni de construir y asignar a los ciudadanos una 'identidad' cultural." (CASTRO-GÓMEZ, 2000, p. 147). 
de tratar de redefinir y acotar su objeto de estudio. Las deliberaciones concluyen con la confección de la célebre Lista Tipo ${ }^{4}$, elaborada bajo una fuerte influencia del pensamiento anglosajón. Si bien ella constituye tan sólo una mera enumeración pragmática de temas, entre los que se encuentra la política educacional, sigue siendo a pesar de los sesenta años de su origen un eje de referencia para la elaboración de marcos teóricos y la confección de currículas universitarias en relación a las ciencias políticas, incluyendo el área curricular de la política educacional actual en las carreras de pedagogía y ciencias de la educación.

Es a partir de 1950 que se comienzan a desarrollar en distintas universidades latinoamericanas las ciencias políticas como campo de estudio con pretensión científica, y casi exclusivamente desde el enfoque jurídico-institucionalista. Esta particularidad hace que la formación en política educacional se centrara durante un largo período sobre la enseñanza de la legislación.

En este marco debemos señalar nuestro posicionamiento respecto al territorio del cual proviene el campo de la política educacional, considerando que en Latinoamérica tuvo un alto impacto la corriente francesa de las ciencias de la educación como campo multidisciplinar pero fuertemente centrado en la pedagogía. A esta matriz se le suma la corriente anglosajona con anclaje en la psicología experimental y la sociología funcionalista (SOUTHWELL, 2003) que da como resultado un campo de la política educacional con un fuerte esquema intervencionista, pragmático y positivista. Los defensores de la corriente francesa - en ocasiones sin saberlo - definen los campos de estudio de las ciencias de

4 Los distintos temas propuestos quedan agrupados en cuatro secciones:

I. Teoría política: a) Teoría política. b) Historia de las ideas políticas.

II. Instituciones políticas: a) Constitución. b) Gobierno central. c) Gobierno regional y local. d) Administración pública. e) Funciones económicas y sociales del gobierno. f) políticas educacionales g) Instituciones políticas comparadas.

III. Partidos, grupos y opinión pública: a) Partido político. b) Grupos y asociaciones. c) Participación del ciudadano en el Gobierno y la Administración. d) Opinión pública.

IV. Relaciones internacionales: a) Política internacional. b) Organización y administración internacional. (UNESCO, 1948). 
la educación como dependientes de la pedagogía. Nuestra perspectiva es otra respecto a la política educacional, dado que las matrices teóricas de la política educacional devienen de las ciencias políticas y se incorporan a las ciencias de la educación donde abreva de la pedagogía. Sin embargo sino se analiza su surgimiento histórico se concluye rápidamente que la política educacional debe centrarse y abrevar de la pedagogía, generando, así, una pedagogización de la política educacional.

También existe otra posición, por ejemplo Jiménez Eguizábal et al. (2006, p. 255) plantea que, "Como forma de conocimiento pedagógico, la política educativa se nos brinda como una nueva atalaya desde la que acceder al conocimiento y a la comprensión del fenómeno pedagógico".

Así se establece el debate de la pertenencia del campo de la política educacional, asumida como campo de la pedagogía y por tanto en la corriente francesa de la filosofía, como campo de las ciencias políticas o espacio autónomo que contribuye a la reflexión de las ciencias políticas y la pedagogía.

Consideramos que aquí se encuentra uno de los ejes que queremos plantear en este artículo: pensar, que en esta diversidad de pertenencias se generan caminos diversos para la enseñanza y formación en política educacional. Con esto argüimos que existen diferencias en la cotidianeidad de las aulas, si partimos de una concepción pedagógica de la política educacional, o una concepción politológica de la política educacional, o finalmente, una concepción autónoma del campo.

Estas disquisiciones no son menores al momento de la enseñanza y menos aún inocuas para quienes poseen ese rol en las universidades. Una cuestión problemática que podemos traer al análisis, tiene que ver con que, aquellos docentes, que asumen este rol, -en ocasiones- no se han planteado para sí mismos, desde cuál de estas posiciones enseñan y forman en la política educacional. 


\section{La matriz histórica de la institucionalización del campo de la política educacional y las epistemes de época}

La institucionalización del campo de las políticas educacionales, con el surgimiento de cátedras de Política Educacional, se debe comprender en el marco del surgimiento de las nuevas carreras de ciencias de la educación en Latinoamérica ${ }^{5}$ con cierta sistematización para la enseñanza y la investigación, en sus diversas versiones: Política Educacional, Política y Legislación Escolar, Política y Educación Comparada, Política y Organización escolar y Política y Administración de la educación. Considerando el intercambio de nombres para las diversas cátedras entre 1950 y 1970.

Es necesario señalar que en las políticas públicas de la década de 1950 se plantea un nuevo modo de desarrollo en Latinoamérica (GOROSTIAGA; TELLO; ÍSOLA, 2012). Con el despliegue de las políticas de bienestar, los organismos de gobierno apelaron a los investigadores en ciencias sociales en busca de soluciones a los problemas que suponía la planificación de las actividades estatales, incluyendo las relacionadas con el crecimiento y reforma de los sistemas educativos. Generando cambios que impactan sobre las nuevas carreras de ciencias de la educación y que fortalecieron la presencia y continuidad de las cátedras - mencionadas anteriormente - en las currículas universitarias. En este devenir de las políticas públicas se observa el surgimiento de la corriente del desarrollismo latinoamericano, y así los perfiles y desarrollo profesional de los graduados en pedagogía o ciencias de la educación se mueven al ritmo del desarrollismo latinoamericano. Considerando que en esta corriente se requería, desde una perspectiva técnica, ejecutores

5 Sobre el giro que tomaron las carreras de Pedagogía hacia Ciencias de la Educación o las nuevas perspectivas que se desarrollaron al interior de las mismas Cfr. Para Argentina: Suásnabar (2009); Southwell (2003); Carlino (1993). Para México: Marín Marín (1997); Pontón Ramos (2002). Para Brasil: Saviani (2007). Para Chile: Nuñez (2002); Cox; Gysling (1990); Avalos (2002). Para Colombia: Restrepo (1983); Jimenez Becerra; Figueroa (2002). 
de políticas educacional, analistas de la legislación escolar, planificadores educativos, técnicos, y analistas estadísticos en educación, entra otras funciones y, desde una perspectiva teórica: la vinculación con los países desarrollados, la formación de recursos humanos y la formación profesional que permitiera identificar - en términos de ese período - las dificultades del subdesarrollo latinoamericano. Según Rama estas nuevas orientaciones tuvieron su principal origen "[...] en los centros especializados en problemas de planificación económica, con una rápida difusión al contar con el apoyo de la planificación educativa." (RAMA, 1984, p. 11).

En el marco de esta re-orientación técnica, teórica y epistemológica, desde fines de los 50 y hasta comienzo de 1970, las cátedras de política educacional comienzan a surgir con una preocupación del valor de lo que está en juego: el análisis teórico y desarrollo de competencias de los graduados en pedagogía o ciencias de la educación para la planificación, la comprensión de la legislación escolar, políticas de universalización de la educación primaria y secundaria. En fin, la investigación educativa, y así el campo de las políticas educacionales, recibieron en la región un fuerte impulso como consecuencia del establecimiento de carreras universitarias en educación. Los estudios conducidos en esa época “[...] consistieron fundamentalmente en análisis descriptivos o estadísticos, para determinar sectores de población donde había que desarrollar la educación primaria o secundaria" (NAZIF; ROJAS, 1998, p. 3). Y para eso se formaba investigadores en políticas educacionales con un fuerte talante desarrollista.

Desde su surgimiento, el campo de la política educacional atravesó vaivenes de corrientes teóricas, filosóficas e interrupciones de política democrática en Latinoamérica, de ideas y conceptos que la estructuran y que se han transformado con los cambios científicos y sociales de la propia realidad educativa, experimentando de este modo, transformaciones que presentan tanto rupturas como continuidades. Es por esto que creemos que los escenarios y desafíos de formación en el campo se comprenderán de forma más acabada si se la contempla desde su proceso histórico. Con esto queremos decir, las epistemologías de la 
política educacional que en la actualidad se pueden crear, serán distintas a la del surgimiento del campo y en consonancia, a las que se puedan plantear en los próximos veinte años, porque las epistemologías se conforman en los contextos culturales, en una fuente de ideas, en un determinado tipo de relaciones teóricas. De este modo nos encontramos ante el desafío, como plantea Giroux (1990), de formar para la incertidumbre de los futuros escenarios.

De este modo, podemos conjeturar que las epistemologías de la política educativa que se nos presentan no serán las de antaño ni las venideras, es allí donde debemos estar atentos a que la formación en política educacional cumplan con su condición ontológica: captar, nombrar, comprender y/o describir la realidad socio-político-educativa. Por tanto preguntas que se suponían contestadas acabadamente en determinado período es necesario volver a estudiarlas a luz de los nuevos interrogantes que se redimensionan ante la realidad y el despliegue teórico que se va desarrollando en el campo.

Por eso es necesario, al menos mencionar, lo que consideramos algunas notas históricas del campo que denominaremos etapas, sólo de un modo provisorio, no intentaremos realizar una estructuración cronológica rigurosa. Dado que toda cronología que intente desplegar la historia del campo de la política educativa puede adolecer de imprecisión, por el sólo hecho de entender la historia como un proceso dinámico, superpuesto e hilvanado.

La primer etapa que denominamos como la política educativa y el enfoque jurídico-institucionalista se caracterizó porque su objeto de estudio se centraba principalmente en el análisis de la normativa legal, en este sentido y tomando la perspectiva de Barrientos del Monte (2009) llevó a que los abogados y especialistas en jurisprudencia fueran durante un largo período, hasta 1960 en Latinoamérica, los responsables autorizados de reflexionar sobre el Estado y las políticas públicas. Esto se observa claramente en las cátedras de política y legislación escolar que se incluyen en los planes de estudios universitarios. La segunda etapa, que ubicamos en los inicios de la década de 1960 y que denominamos 
como la política educativa en la perspectiva de la planificación desarrollista tuvo su base en cuanto a perspectiva teórica en conjunto con el proceso de institucionalización y fortalecimiento de las ciencias en la región a través de la creación de oficinas estatales, institutos de investigación y organismos regionales de planificación (CALDERÓN; PROVOSTE, 1992; DE SIERRA et al., 2006). Es en estas décadas que podríamos hablar del período de protagonismo de la economía, la sociología y la ciencia política en los ámbitos académicos dado que se presenta la demanda por parte del Estado de estos campos, como hemos mencionado anteriormente. Es así que en la década de 1960 podemos observar que el campo de la política educativa está, fuertemente vinculada a la teoría de la planificación y el planeamiento educativo, con el auge del desarrollismo y la teoría del capital humano en Latinoamérica (SUÁSNABAR, 2009). Es el período de la política educativa que se desarrolla desde el enfoque de la planificación que dirigía la mirada "[...] a la eficiencia interna (rendimiento cuantitativo) y cuál es su eficacia (adecuación en número y tipo de egresados a las necesidades de mano de obra)" (PAVIGLIANITTI, 1996, p. 8). Desarrollándose la Sociología de la Educación bajo el paradigma funcionalista y el surgimiento de la Economía de la Educación que se situaría en el análisis del desarrollismo y el capital humano.

La tercera etapa, hacia fines de la década de 1960 y durante la década de 1970 que denominamos la política educativa y la perspectiva crítica, es la del surgimiento de las miradas y enfoques críticosreproductivistas que comenzaban a cuestionar el funcionamiento de los sistemas educativos nacionales, dando lugar a los estudios de etnografía educativa dentro de las políticas educativas, al desarrollo de la sociología política crítica, y a perspectivas neo-marxistas y pos-estructuralistas entre otras.

Su contribución principal consistió en quebrar la mirada optimista, de orden lineal y progresivo de crecimiento, construida por las clases dominantes en las décadas del cincuenta y del sesenta. Además, pusieron de relieve las formas y los mecanismos de la desigualdad social que están incorporados en los sistemas educativos, sacaron a la 
luz sus conexiones con las diferencias culturales, con la reproducción de la mano de obra y con la consolidación de las relaciones sociales y políticas. (PAVIGLIANITTI, 1996, p. 4).

La década de 1970 encuentra a los países de Latinoamérica cruzados por dictaduras feroces que paralizaron el desarrollo del campo, particularmente de las últimas concepciones neo-marxistas. Comienzan a desenvolverse enfoques de política educativa como la educación como restauradora del orden, del orden natural, del personalismo y del estudio de los agentes de la educación (PUIGGRÓS, 1986).

A medida que fueron finalizando los gobiernos dictatoriales en Latinoamérica comenzó a emerger el optimismo pedagógico abriendo los caminos de investigación hacia el paradigma de educación y democracia (SUASNÁBAR, 2009), expresado generalmente en la perspectiva de educación y sociedad. Así, la cuarta etapa se da con la instauración de las democracias latinoamericanas, pasando por los enfoques de Política $y$ administración de la educación y luego influenciada por el neoliberalismo a política y gestión de la educación en sus dos vertientes, la vinculada al managment y la que lo hace desde una perspectiva humanista o antihegémonica (MIÑANA BLASCO, 2003).

Cuando se introdujo la temática de gestión educativa en Latinoamérica se la asociaba, desde las perspectivas críticas, a una visión liberal-capitalista que intentaba evaluar el fenómeno educativo en términos de costo-beneficio de recursos humanos, en fin como una vuelta, aunque más pronunciada y sofisticada, al desarrollismo de la década de 1960. Ahora bien, de modo simultáneo emerge la categoría temática de gestión educativa, como campo disciplinar ${ }^{6}$, que data de los años sesenta en Estados Unidos, de los años setenta en el Reino Unido y de los años ochenta en América Latina (CASASSUS, 2000).

6 Desde nuestra perspectiva la categoría de campo disciplinar no es equivalente a disciplina, sino a campo teórico. 
Como hemos visto existen epistemes de época para cada período histórico, éstas son múltiples y diversas. Esto nos indica que no existe un objeto de estudio de la política educacional y en consecuencia una perspectiva epistemológica a seguir para formar a los jóvenes investigadores porque la episteme de época exigirá del joven investigador en unos 30 años, y ante un nuevo escenario, responder con diversas categorías analíticas, quizá desconocidas hasta hoy.

\section{La crisis de las ciencias sociales y políticas: crisis de la política educacional}

En 1958, Braudel escribía Hay una crisis de las ciencias del hombre, esta aseveración se mantuvo y expresó de diversos modos en los últimos años. Existen múltiples razones por las cuales podríamos elucubrar que las ciencias sociales ingresaron en un período de crisis, pero principalmente podemos considerar que el mundo social - para denominarlo desde un lugar amplio - se ha desplegado con vertiginosos cambios y transformaciones aceleradas, y así las diversas categorías de las ciencias sociales fueron quedando al margen de la potencialidad de miradas que le permitan comprender la realidad social.

Estamos atravesando una etapa de profundos cambios sociales, con realidades cada vez más complejas de ser abordadas por las estructuras conceptuales convencionales, lo cual conlleva a que la realidad de los fenómenos que se nos presentan a los investigadores en ciencias sociales, carezcan de referentes conceptuales y viceversa. Las estructuras y categorías conceptuales con las que nombrábamos de algún modo lo que acontecía socialmente carecen de potencialidad explicativa. En fin, estamos viviendo la revolución contemporánea del saber y la complejidad social (véase SOTOLONGO CODINA; DELGADO DÍAZ, 2006). Ante esta situación Wallerstein (1999) plantea que las ciencias sociales tiene grandes limitaciones en el estudio de las realidades sociales por lo cual habría que pensar nuevos fundamentos epistemológicos, para esto propone un desafío: impensar las ciencias sociales que se opone a la categoría de re 
pensarla, dado que re pensarlas se convertiría en una barrera epistémica para la creación de nuevas epistemologías. Así comienzan a emerger - a nivel internacional y latinoamericano - algunas perspectivas analíticas de la realidad social como pensamiento alternativo (VERGARA ESTÉVEZ, 1991).

En el mismo sentido, las ciencias políticas se enfrentan a interrogantes similares, por ejemplo siguiendo los postulados de Giovanni Sartori (2004) en un reconocido artículo denominado ¿Dónde está la ciencia Política?; César Cansino en el año 2006 (y luego en el 2008) anuncia el adiós a la ciencia como la crónica de una muerte anunciada con muchos debates a su alrededor ${ }^{7}$ Explicando que "[...] terminó alejándose del pensamiento y la reflexión, hasta hacer de esta ciencia un elefante blanco gigantesco, repleto de datos, pero sin ideas, ni sustancia, atrapada en saberes inútiles para aproximarse a la complejidad del mundo." (CANSINO, 2006, p. 13). En el mismo eje de reflexión De Sierra et al. (2006, p. 51) señalan que en América Latina en los últimos años se debilita en las ciencias sociales la dimensión critico-utópica afirmando que "[...] hay menos reflexión sobre la disciplina y más sobre el uso que la sociedad hace del conocimiento de las ciencias sociales”. Por su parte, Atilio Borón advierte que es necesario darse "[...] una minuciosa discusión sobre algunos de los principales problemas que plantea la necesaria reconstrucción de las ciencias sociales sobre nuevas bases epistemológicas" (BORÓN, 2006, p. 16).

La realidad de las ciencias sociales y políticas y en particular de la política educacional requiere una transformación epistemológica que permita construir y desplegar nuevas epistemologías para repensarla, a partir del atrevimiento científico que, de algún modo, resquebraje y sacuda las perspectivas epistemológicas actuales, lo cual permitirá - a partir de esa deconstrucción - un espacio para iniciar un nuevo

7 La fuerte crítica a la ciencia política, que tiene pretensiones hegemónicas, provocó la discusión, la controversia y la polémica. Prueba de ello son los trabajos de Negretto (2004); Colomer (2004); Laitin (2004); Oxhron (2006); Covarrubias (2006) y Lucca (2008) que, de una u otra forma, presentan posiciones en el marco de la polémica. 
camino epistemológico en Latinoamérica. Abandonando los caparazones procedimentales y asumiendo miradas complejas capaces de abordar y analizar las nuevas realidades socio-educativas.

\section{La enseñanza de la política educacional y la formación de investigadores en el campo}

En la enseñanza de la política educacional y la formación de investigadores en el campo suele existir una dificultad que es la ausencia del posicionamiento epistemológico del formador, es decir, en ocasiones se presentan libros o artículos para la explicación de un concepto o categoría sin plantear desde dónde se lo está haciendo, y sino se plantea desde que posicionamiento se está intentando comprender la categoría o concepto se presenta como único y verdadero. Así, argüimos que para comprender las categorías y conceptos del campo de la política educacional es clave desarrollar como primer paso conceptual: comprender las perspectivas y posicionamientos epistemológicos de desarrollo para el análisis y comprensión de las políticas educativas. Esto implica comprender desde dónde se observa, desde donde se hace el análisis y por lo tanto qué se incluye y qué se excluye en el análisis en términos de perspectiva epistemológica. Dado que en términos teóricos no existirá la definición, sino definiciones desde diversas miradas.

Esto es clave, porque el politólogo de la educación analiza conceptos siempre nuevos en las complejas relaciones sociales que se establecen. Por tanto, explicar y comprender la reforma educativa, leyes, etc. que están aconteciendo en un determinado país y momento histórico es necesario para el proceso de formación pero en ocasiones no se explican modelos analíticos para comprender las reformas, leyes, etc. o tratando de ser más claro en nuestro posicionamiento: no se despliegan diversos modelos analíticos para comprender las políticas educativas. Al menos los modelos analíticos conocidos hasta ese momento en el cual se produce el proceso de la formación. Por caso: los modelos de análisis de políticas públicas de Lasswell (1951); de la interacción política 
(LINDBLOM, 1959); de la teoría sistémica (EASTON, 1976); policy cycle (JONES, 1970); de las redes y de las comunidades políticas (KRIESI, 1994; SABATIER; JENKINS-SMITH, 1999, 1988); el modelo de advocacy coalitions (SABATIER; JENKINS-SMITH, 1999, 1988); abordaje cognitivo de políticas públicas (MULLER, 1990, 2000; MULLER; SUREL, 1998, 2002); y así podríamos seguir con varios modelos de análisis, por ejemplo el top down y bottom up, enfoque institucionalista y neo-institucionalista, entre otros. La pregunta: ¿estos modelos se desarrollan y explican en los procesos de formación de los investigadores en política educacional?

La dificultad radica en que si estos u otros modelos no se estudian, analizan y reflexionan aquel joven que se está formando como investigador queda atrapado entre la lógica de las matrices históricas, el posicionamiento epistemológico del investigador, la episteme de época o alguna metodología de moda.

Esto es, el eje de la formación es la lógica y la epistemología del conocimiento en política educativa. Alguna vez una colega me dijo que preocuparse de esas cuestiones tan teóricas no era política educativa, sino perder el tiempo, y que el desafío consistía en que desde las aulas y en la formación de investigadores meterse en la realidad. Claro que estoy absolutamente de acuerdo con mi colega, pero al desarrollar un proceso de formación basado en la coyuntura, en la realidad actual, en las disquisiciones de los debates de los medios de comunicación sucedían dos cosas:

a) En primer lugar se perdía capacidad analítica como politólogo de la educación. Llegando por momento a que sus estudiantes opinen desde un mero sentido común ${ }^{8}$.

b) Imposibilitaba a sus estudiantes formarse como futuros investigadores en política educacional. Claramente ellos no poseían capacidad analítica. Y aquellos que lograban otro tipo de miradas

8 Entendemos la categoría de sentido común como aquella que es fuertemente influenciada por las representaciones sociales, creencias y juicios que no permiten un análisis riguroso de un escenario o situación social. Considerando que la rigurosidad no la entendemos como neutralidad, sino como capacidad analítica para comprender. 
se debían solamente al esfuerzo del propio alumno o al encuentro con otro formador que lo orientaba en cuestiones de análisis de política educacional.

El modelo que el investigador posee sobre las políticas educacionales surge de un posicionamiento epistemológico (TELLO, 2012; TELLO; MAINARDES, 2015b). Dependiendo de la concepción que tiene el investigador se inclinará por determinada construcción de su objeto de estudio para la investigación en política educacional. En ocasiones por no haber estudiado y reflexionado sobre esos modelos los jóvenes investigadores creen que existe una definición de política educacional, sin descubrir las múltiples definiciones de política educacional. Las concepciones que posee el investigador en forma de modelizaciones teóricas devienen de la propia formación del investigador, de su construcción subjetiva y de las producciones teóricas con las que se ha vinculado En este sentido es clave, para quienes nos desempeñamos como formadores de investigadores en política educacional, mostrar la mayor cantidad posible de modelos teóricos analíticos para la reflexión y análisis profundo de los estudiantes. En ocasiones puede suceder que los investigadores formados no estemos de acuerdo con algunos modelos, pero es necesario que los estudiantes los conozcan y estudien, y reflexionen sobre ellos.

Aquí también se debe considerar cuál es el objetivo de la formación en política educacional y que pueden ser legítimos cualquiera de ellos, pero se debe ser consciente que eso implica diversos niveles de abstracción y desarrollo teórico:

a) formar para el interés y la preocupación por los asuntos de política educativa (en términos de ROIZ, 1982, sujetos con consciencia política).

b) Formar para la investigación analítica en política educacional.

En sentido, Nohlen al referirse a la formación en ciencias políticas plantea que existe un área clave en la formación: 
[...] la constituye la teoría política, que incluye tres campos: a) la historia de las ideas o la filosofía política, b) la teoría política moderna, o sea las grandes corrientes teóricas, las de gran alcance y las metateorías o los paradigmas científicos y, finalmente, c) la metodología en ciencias sociales. La importancia de esta área tan heterogénea en la enseñanza, en su contribución al desarrollo del conocimiento de los fundamentos de la política en cuanto a lo normativo y lo teórico, por un lado, y lo metodológico en el estudio de la política, por el otro (2003, p. 5).

Ahora bien, en cualquiera de los dos casos, el interés y la investigación, requieren de aparatos conceptuales, modelos analíticos y esquemas teóricos para su comprensión. Como dijimos, quizá, con distintos niveles de complejidad.

Esto se puede observar en las tesis de maestría y doctorado, $y$ en ocasiones en algunas investigaciones de investigadores formados cuando se observan desarrollos monocausales y lineales en los procesos de análisis. En este sentido Anderson y Scott (2013, p. 528) al referirse a la investigación en políticas educacionales en EE. UU plantea que "[...] muchas investigaciones cualitativas - por lo menos en USA - poseen un fuerte sesgo a favor de interpretaciones y atribuciones de 'causalidad' sin desplegar ningún análisis". O por otro lado trabajos e investigaciones con un fuerte sentido de descripción. En este sentido es necesario considerar, siguiendo el planteo de Mainardes (2015) que la descripción es parte de la investigación y no la rechazamos per se. Ahora, la dificultad radica cuando no se profundiza en los niveles de investigación y la descripción se convierte en descripcionismo como único paso del proceso de investigación. $\mathrm{Y}$ esto puede suceder porque fue el componente central de su proceso formativo como estudiante de grado y posgrado. Los formadores contaron, narraron, describieron las políticas educacionales. Como hemos observado en una investigación reciente (TELLO; MAINARDES, 2012) sobre posicionamiento y perspectivas epistemológicas en política educacional:

Otra de las carencias apreciadas en el relevamiento realizado residió en la ausencia del posicionamiento epistemológico del investigador 
de la investigación y, en algunos casos, la inclusión de matrices epistemológicas que generaban investigaciones con poca consistencia teórica en su análisis y resultados. (TELLO; MAINARDES, 2012, p. 5).

Un error común que se comete en la formación de investigadores en políticas educacionales es creer que la mera incorporación a un equipo de investigación será de utilidad para el estudiante. Sin embargo, en ocasiones, no se tiene en cuenta que allí se aprende el oficio de investigador es decir, aprender del profesor con más años de experiencia, sin embargo suele suceder que en la expertise del profesor e investigador formado no se hacen explícitos los procedimientos o los modelos teóricos empleados para investigar, dejando a un lado los posicionamientos epistemológicos, las perspectivas epistemológica y enfoques epistemetodológicos con los que se desarrolla la investigación.

En este sentido, se debe considerar el investigador formado ya ha construido estas categorías y posiblemente las ha trabajado durante largo tiempo, sin embargo el estudiante las tomará. Solo eso: las tomará, y en realidad debe hacer su propio proceso de construcción mientras participa de un equipo de investigación. En este sentido el responsable de la formación tendrá el desafío de pensar en estas cuestiones respecto a sus estudiantes.

\section{El sentido de la formación en política educacional y las polity, politics y policy}

La primera aclaración conceptual que es necesario plantear en este apartado la hacemos a fines de distinguir los estudios sobre la Política Educacional (lo que denominamos campo teórico o epistemologías de la política educacional), y las Políticas Educacionales (aquellas que hacen referencia específica a la gestión, toma de decisiones y acción política). Max Weber (1991, p. 83 [1918]) en El político y el científico, sostuvo con énfasis la clara diferenciación que distingue a "[...] las tomas de posición 
política" (Políticas educacionales en plural) del "análisis científico de los fenómenos políticos" (Política Educacional en singular).

En ocasiones se comete el error de definir el campo de la política educacional vinculada a adjetivaciones o a la realidad política. Y esta dificultad es una carga que tenemos los investigadores latinoamericanos de la escuela americana con una definición muy empleada en nuestras investigaciones. Nos referimos aquí a las politics y a las policy, que para los no anglo-parlantes son categorías difusas y en ocasiones con alguno de estos términos se intenta nombrar el campo teórico. Yehezkel Dror (1982, p. 33) afirma que "[...] la falta de una diferencia entre 'politics' y 'policy' en un gran número de idiomas, crea un gran problema en el desarrollo del análisis de políticas en muchos países. Al mismo tiempo, la falta de esta diferencia señala realidades y percepciones descuidadas en el análisis de políticas [...]" (el énfasis es mío).

Siguiendo los postulados de Nohlen (2003, p. 4) "[...] las tres dimensiones de la política que, debido a limitaciones idiomáticas, ni en alemán ni en castellano podemos diferenciar conceptualmente bien: polity, politics y policy (en inglés), o sea la forma, el proceso y el contenido de la política". En este sentido el autor plantea un ejemplo refiriéndose a la cuestión de la democracia y explica que para conocer la categoría de democracia se deben atravesar tres momentos: como forma (polity) y proceso (politics), y también se deben analizar las condiciones internas de las democracias como resultado (policy). Aquí debemos comprender inicialmente que ninguna de las tres designa el campo teórico y epistemológico de la política educacional. A diferencia de lo que podría suceder si mencionamos las ciencias políticas, en su propia categorización de ciencias pareciera encontrarse el trasfondo teórico y epistemológico de las ciencias políticas y de la cual abreva la política educacional.

Siguiendo a Ana Vitar (2006, p. 26) "No debemos confundir los procesos políticos y sociales con las herramientas para pensarlos". Así podemos afirmar que en términos de la academia americana nos estaríamos refiriendo a la policy educational science. Más allá de que la consideremos una ciencia o no. 
En tiempos de no demarcacionismo epistemológico ${ }^{9}$ no considero necesario tener que demostrar que la política educacional es una ciencia o una disciplina. Más bien prefiero denominarla con un campo de estudios. Y, esto nos lleva a comprender como campo teórico, y ahora sí, si se quiere, también como: polity, politics y policy. El desarrollo que estamos planteando aquí nos centra en el campo teórico de la formación para poder comprender la forma, el proceso y los resultados de las políticas educacionales. Pero sin formación en el campo teórico y epistemológico, esto es, lo que hemos denominado en otro lado "[...] las epistemologías de la política educativa” (TELLO, 2012, p. 57) sería casi imposible abordar ese análisis.

Por otro lado existe una concepción del sentido común de la política o las políticas. Refiriéndonos ya, a las acciones de gobierno. Y es allí donde encontramos múltiples opiniones sobre esas acciones. Sin embargo aquellos que se forman en la investigación en políticas educacionales requieren desnaturalizar-se del sentido común, para lograr abordajes analíticos de la realidad. O como plantean Muller y Surel (1998) es preciso señalar que la política pública no existe naturalmente en la realidad, sino que se trata a la vez de una construcción social y de una construcción de un objeto de estudio. Esto implica dificultades para la identificación de sus contornos exactos, en este sentido las modelizaciones nos permiten llevar a cabo ejercicios heurísticos para comprender la hechura de la política educacional.

En este sentido es clave asumir el posicionamiento de Ball, cuando afirma:

9 No podríamos considerar que es posible definir la teoría de la política educacional, ya que no haríamos otra cosa que una reducción epistemológica, dado que los planteos epistemológicos actuales no requieren de la demarcación disciplinar, lo cual responde más bien a estudios y concepciones clásicas desde la epistemología que distinguía por ejemplo: Ciencia-PseudocienciaDisciplinas, entrando en el falso camino del cientificismo; por el contrario, asumimos en el sentido de Wallerstein (1999), una postura a favor de la ciencia y el conocimiento y en contra del cientificismo. 
[...] El significado de la política se da por sentado teórica y epistemológicamente integrándose en las estructuras analíticas que ellos construyen. No es difícil encontrar que el término política se utiliza para describir muy diferentes 'cosas' en diferentes puntos en el mismo estudio. Para mi mucho se apoya en el significado o posibles significados que damos a la política; esto afecta 'cómo' investigamos y cómo interpretamos lo que encontramos (BALL, 1994, p. 15).

Ahora bien, es necesario considerar que las múltiples significaciones vienen de los trabajos que han planteado en su desarrollo epistemológico e histórico las ciencias políticas, a través de diversos referentes teóricos y que ninguna de ella posee un carácter neutral o totalmente explicativo y menos aún verdadero. Sin embargo, esas significaciones que en ocasiones se convierten en modelizaciones impactan sobre la construcción subjetiva en la formación del investigador en política educacional.

Estas modelizaciones se proponen explicar el surgimiento, el cambio o el desarrollo de las políticas públicas, comprendiendo que el trabajo científico consiste, en gran parte, en proponer teorías para explicar fenómenos, en nuestro caso específico de las políticas educacionales.

Así nos preguntamos: ¿tenemos claridad sobre la diferencia entre polity, politics y policy? O las empleamos como sinónimos de políticas educacionales. Cómo transmitimos estas ideas a los jóvenes investigadores? ¿Cuándo hablamos de política educacional todos los docentes y formadores de investigadores comprendemos lo mismo?

\section{De las metodologías a las epistemetodologías para la enseñanza y formación de investigadores en política educacional}

Existe aún en la enseñanza de las metodologías de la investigación en política educacional - tanto en la enseñanza como en la formación de investigadores - una concepción instrumentalista y positivista de los métodos empleados en política educacional. Donde se observa una ausencia de reflexión epistemológica sobre las metodologías 
que se enseñan o se emplean en la investigación en política educacional. Como si la presencia del método ofreciera rigurosidad científica por sí sola. Y como hemos planteado en otro lado (TELLO; MAINARDES, 2012) donde se realizó una metainvestigación sobre un grupo de producciones publicadas en revista latinoamericanas observamos que: "[...] en el conjunto de las investigaciones se detectó que el uso de los referenciales teóricos se planteaba en términos metodológicos, aislados de los fundamentos epistemológicos con los que fueron elaborados" (TELLO; MAINARDES, 2012, p. 2).

Como dijimos la metodología en sí misma no se identifica con la perspectiva epistemológica. En todo caso lo hace una epistemetodología (TELLO, 2011, p. 7) que es "[...] la conjugación de la metodología con el posicionamiento epistemológico del investigador en el desarrollo de proceso de producción de conocimiento."

Con epistemetodología nos referimos al momento metodológico donde el investigador opta por una u otra metodología. No consideramos a los enfoques metodológicos como meros instrumentos sea de recopilación sea de análisis de la información sino como el método del logos, esto es el modo de pensar el logos. Por tal razón preferimos hablar de epistemetodología, categoría en la que confluyen la presentación de método y la posición epistemológica del investigador. Consideramos que un enfoque metodológico posee una epistemología, pero a los efectos de la distinción de uso común, preferimos este último término: epistemetodología. Pero no por una cuestión de mera sofisticación en los conceptos, sino más bien de precisión. Es decir, debemos tener en cuenta que la metodología se convierte en una epistemetodología en la medida que posee un sustento teórico y epistemológico. Por sí sola y, por ejemplo a los efectos de la enseñanza son sólo metodologías, sin embargo son metodologías que muchas veces se enseñan como epistemologías, Bourdieu es agudamente irónico respecto de esta predominancia de una idea instrumental de metodología que la concibe como "[...] serie de recetas o de preceptos que hay que respetar no para conocer el objeto sino para ser reconocido como conocedor del objeto." (BOURDIEU, 1997, p. 62). Esto es, para dar un maquillaje de cientificidad. 
En síntesis, la cuestión que presentamos no es si la investigación y la proyección social que realizan las ciencias humanas y sociales contienen o no valores e ideología, sino la necesidad de indagar qué valores concretos concurren en cada proceso y situación, cuál es su papel y cómo se podrán, y deberán -desde las diferentes visiones de realidad-, manejar e integrar en la práctica. Un principio para tener en cuenta es que no se puede eliminar la subjetividad y los valores de la ciencia y de sus usos técnicos; habría, en cambio, que esforzarse en hacerlos explícitos para observar su aportación al resultado final de la actividad de investigación en política educacional.

Con esta orientación, el investigador asume mayor libertad de elección de un curso de acción o de un método determinado pero, paralelamente, mayor es la responsabilidad (personal y profesional) por las consecuencias de su proceder y por el valor social de los resultados de sus investigaciones, en tanto posición política: “¿hasta qué punto ese conocimiento contribuye o no a la posibilidad de construir una sociedad más democrática y más equitativa?" (LANDER, 2000, p. 54).

El pasaje de enseñar y formar en metodologías a las epistemetodologías en política educacional se convierte en un desafío para los espacios académicos invadidos de positivismo e instrumentalismo cientificista más preocupado por las formas que por la producción del conocimiento.

Hemos podido observar como la cuestión metodológica se simplificó a los efectos de la enseñanza, escindiéndola de su sustento: la epistemología. Así, en los talleres de metodología de la carreras de grado y posgrado en Latinoamérica se re afirman técnicas metodológicas que prescinden de perspectivas epistemológicas extendiéndose a posteriori en las investigaciones políticas educacionales (Cfr. TELLO, 2011; BARRIGA; HENRÍQUEZ, 2003).

Ahora bien, debemos observar lo que acontece en niveles básicos de la investigación en política educacional - a veces dejados de lado es necesario volver a plantear un principio fundamental: la investigación no es neutra, no está posicionada en ningún lado, por el contrario 
el investigador construye, particularmente en política educacional, conocimientos desde una posición epistemológica. Aquí debemos considerar el planteo de Ball (2006) cuando se refiere a la investigación en políticas educacionales señalando por un lado la estructura de una investigación a la que él denomina epistemología de superficie como la relación que se establece entre conceptualizaciones, conducción, diseño y desarrollo de una investigación. Es decir, allí se encuentra un modo de hacer epistemología, pero no puede dejar de tenerse en cuenta para la solidez y consistencia de la misma lo que el autor denomina epistemología profunda, esto es las formas de interconexión teórica, lógica y epistemológica entre los componentes de una investigación en política educacional.

Teniendo en cuenta estas consideraciones podríamos pensar que existen una epistemología de Nivel I y otra de Nivel II, es decir a modo de pasos que se presentan en una investigación. Pasos inherentes que se retroalimentan a medida que se avanza en cada uno de los niveles, por ejemplo, el diseño de la investigación no es una tarea meramente técnica ni ausente de posicionamiento epistemológico. Y como dijimos una investigación se sustenta desde sus inicios (el diseño, los objetivos, la construcción del objeto, entre otros) desde una determinada visión del mundo. Sería ingenuo considerar a alguno de estos niveles, principalmente al nivel I como ausente de posicionamiento epistemológico. La dificultad se encontraría siguiendo los postulados de Ball (2006) en cuanto que muchas investigaciones se llevan a cabo desde lo que nosotros llamamos nivel I, es decir,muchas investigaciones en política educacional son investigaciones que sólo desarrollan y explicitan una epistemología de la superficie: la presentación de un tema, un objeto de estudio, marco referencial, metodología, etc. Pero sin conexiones. Lo que da como resultados de investigaciones, en general, descripciones a modo de descripcionismo de la realidad político educacional.

Con esto planteamos - nuevamente - que la presencia de marco teórico y metodología en una investigación, por si mismos, no expresan la epistemología de la política educativa de una investigación, allí estaríamos en el Nivel I de análisis epistemológico. Se podrá 
contra-argumentar que estos se pueden encontrar en forma subyacente, pero allí estamos ante una dificultad epistemológica en términos de Bourdieu, dado que lo no explícito refiere a los no-consciente, explicará el autor. Y sobre lo inconsciente dirá Bourdieu en el oficio del sociólogo es muy difícil reflexionar. Porque el investigador despliega la posición de un modo naturalizado, podríamos decir: sólo para sí mismo.

El nivel II daría cuenta de la presencia de una epistemología de la política educacional. Con esto argüimos, el marco teórico de una investigación es el insumo para hacer análisis o epistemología de la política educativa, ahora bien, llevar a cabo el ejercicio de las epistemologías de la política educativa, es en el sentido de Gewirtz (2007, p. 7), llevar a cabo una "[...] reflexividad ética sobre el trabajo de investigación [...]"; reflexionar sobre las posibles consecuencias éticas de la investigación, de qué forma ese trabajo legitima ciertos posicionamientos y no otros. En fin, la reflexividad ética es el modo que el investigador asume la realidad, en términos de Boaventura Souza Santos (2006, p. 18):

Eso es muy importante, ya que nosotros aprendemos con nuestra epistemología positivista que la ciencia es independiente de la cultura; sin embargo, los presupuestos culturales de la ciencia son muy claros. Por tanto, vamos a discutir cómo podemos, en lo que respecta a la ciencia, ser objetivos pero no neutros; cómo debemos distinguir entre objetividad y neutralidad. Objetividad, porque poseemos metodologías propias de las ciencias sociales para tener un conocimiento que queremos que sea riguroso y que nos defienda de dogmatismos; y al mismo tiempo, vivimos en sociedades muy injustas en relación a las cuales no podemos ser neutrales. Debemos ser capaces de efectuar esta distinción, que es muy importante.

Como señala Galindo Cáceres (1999, p. 17), “[...] para muchos [investigadores] este punto, es decir, la dimensión epistemológica es irrelevante, como si se encontrara fuera del oficio del investigador. McLuhan (1965, p. 18) dice: "No sé quién descubrió el agua por primera vez, pero estoy seguro de que no fueron los peces". En efecto, las personas insertas 
en un proceso naturalizan sus propios posicionamientos sin observar la necesidad de hacerlos explícitos. Y es aquí donde es necesario volver sobre la categoría de Gewirtz (2007), dado que, considera que la reflexividad ética de la producción de conocimiento en política educacional tiene efectos sobre el propio mundo social que el investigador analiza y allí existe la responsabilidad ética de la investigación en tanto la exigencia de explicitar con claridad la posición epistemológica del investigador, es decir llegar a una epistemetodología de la investigación en política educacional.

Aquí existe un gran desafío en la enseñanza y formación de investigadores en política educacional.

\section{Consideraciones finales}

Sin dudas que los formadores de investigadores en política educacional debemos hacer un viraje en nuestros modos de acompañar los procesos formativos de los futuros investigadores. Un proceso formativo que no quede desprendido de las matrices históricas, de la episteme de época y de la realidad actual y sus coyunturas, pero que simultáneamente les permita a los jóvenes investigadores, a partir del conocimiento, generar nuevos esquema analíticos para una realidad política cada vez más compleja y difusa. En términos de Tiramonti (2003, p. 74):

Existe un fenómeno que es importante señalar que emerge de los `90: es el agotamiento de nuestros marcos conceptuales. El instrumental teórico y las metodologías de análisis para analizar el campo de la educación se muestran insuficientes o no aptos para dar cuenta de la complejidad de fenómenos y situaciones que se dan en la realidad [...].

Algunos plantean que la realidad se ha distorsionado. Será que los modelos analíticos han quedado superados por la realidad como plantea la autora? De lo que estamos seguros es que se requiere conocer esos modelos y sentidos de las políticas educaciones para generar nuevos modelos de la compleja realidad. 
Es decir, el vacío teórico implica mayor profundización teórica, conceptual y epistemológica porque en la formación de investigadores no se trata de innovaciones radicales que surgen del simplismo ateórico, sino de una formación que le permita desarrollar nuevos modelos que surjan de los procesos históricos del conocimiento.

Como planteábamos anteriormente, nos preocupa la realidad, y parta atenderla debemos tratar de entenderla. Y porque queremos entenderla debemos consolidar los procesos de enseñanza y formación con solidez.

\section{Referencias}

ANDERSON, G.; SCOTT, J. Investigando las políticas educativas: ¿Cómo teorizamos la "causalidad" entre lo macro y lo micro? In: TELLO, C. (Coord. y Comp.). Epistemologías de la política educativa: posicionamientos, perspectivas y enfoques. Campinas, SP: Mercado de Letras, 2013. p. 527-532.

ANDERSON, G. L. Cambios macropolíticos e institucionales, nuevos paradigmas e impacto en el trabajo docente. In: POGGI, M. (coord.). Políticas docentes. Formación, trabajo y desarrollo profesional. Buenos Aires: IIPE-UNESCO, 2013.

AVALOS, B. Profesores para Chile: historia de un Proyecto. Santiago: Ministerio de Educación, 2002.

BALL, S. J. Sociologia das políticas educacionais e pesquisa crítico-social: uma revisão pessoal das políticas educacionais e da pesquisa em política educacional. Currículo sem Fronteiras, v. 6, n. 2, p. 10-32, jul./dez. 2006.

BALL, S. J. Education reform: a critical post-structural approach. Buckingham: Open University Press, 1994.

BARRIENTOS DEL MONTE, F. "La ciencia política en América Latina. Apuntes para una historia (estructural e intelectual) de la disciplina en la región.", Texto preparado para el Seminario de Investigación del Área de Ciencia Política y de la Administración de la Universidad de Salamanca. Instituto Italiano di Scienze Umane, Florencia, Italia. 5 nov. 2009. Disponible em: <http://campus. usal.es/ dpublico/areacp/ Posgrado2009-2010/Barrientos.pdf>. Acceso em: 24 jul. 2011. 
BARRIGA, O.; HENRÍQUEZ, G. La presentación del objeto de estudio. Revista Cinta de Moebio, Santiago, n. 17, p. 1-20, 2003. Disponible en: <http://www. facso.uchile.cl/publicaciones/ moebio/17/barriga.htm>. Acceso: 24 jul. 2009.

BORÓN, A. Las ciencias sociales en la era neoliberal: entre la academia y el pensamiento crítico, Tareas, Panamá (CELA - Centro de Estudios Latinoamericanos "Justo Arosemena"), n. 122, enero-abril, 2006.

BOURDIEU, P. Capital cultural, escuela y espacio social. México: Siglo XXI, 1997.

BRAUDEL, F. La longue durée, Réseaux, v. 5, n. 27, p. 7- 37, 1958.

CALDERÓN, F.; PROVOSTE, P. Autonomía, estabilidad y renovación. Los desafíos de las ciencias sociales en América Latina. Buenos Aires: CLACSO, 1992.

CANSINO, C. Adiós a la ciencia política - Crónica de una muerte anunciada, Metapolítica, n. 49, p. 26-37, septiembre - octubre, 2006.

CANSINO, C. La muerte de la ciencia política. Buenos Aires: Sudamericana-La Nación. 2008.

CARLINO, F. Transformaciones en la carrera de ciencias de la educación de la UBA. Un recorrido histórico de algunos problemas actuales, Revista del Instituto de Investigación en Ciencias de la Educación, Buenos Aires, Argentina, año II, n. 3, p. 48-58, 1993.

CASASSUS, J. Problemas de la gestión educativa en América Latina. Santiago: UNESCO, 2000.

CASTRO-GÓMEZ, S. (ed.). La reestructuración de las ciencias sociales en América Latina, Bogotá, Instituto PENSAR, p. 49-70, 2000.

COLOMER, J. La ciencia política va hacia adelante (por meandros tortuosos): un comentario a Giovanni Sartori, Revista Política y Gobierno, México, CIDE, v. 9, n. 2, p. 355-359, 2004.

COVARRUBIAS, I. La ciencia política frente al espejo, Metapolítica, n. 49, septiembre-octubre, 2006. 
COX, C.; GYSLING, J. La Formación del Profesorado en Chile 1842-1987. Santiago de Chile: CIDE, 1990.

DE SIERRA, G., et al. Las ciencias sociales en América Latina en una mirada comparativa. In: TRINDADE, H. (Org.). Las ciencias sociales en América Latina en perspectiva comparada. México, DF: Siglo XXI, 2006. p. 17-53.

DROR, Y. Public Policy Making Reexamined. New Brunswick: NJ, 1982.

EASTON, D. Esquema para el análisis político. A Buenos Aires: Morrortu Editores, tercera edición, 1976.

GALINDO CÁCERES, J. Del objeto construido al objeto percibido, Estudios sobre las culturas contemporáneas. Época, Colima, México, v. 5, n. 9, p. 9 -24, 1999.

GEWIRTZ, S. A. reflexividade ética na análise de políticas: conceituação e importância. Práxis Educativa, Ponta Grossa, v. 2 , n. 1 , p. 7-12, jan./jun. 2007.

GIROUX, H. Los profesores como intelectuales. Barcelona: Paidos, 1990.

GOROSTIAGA, J.; TELLO, C.; ISOLA, N. Investigación educativa en América Latina: Notas históricas y tendencias recientes. In: PALAMIDESSI, M.; SUASNÁBAR, C.; GOROSTIAGA, J. (Comp.). Investigación educativa y política en América Latina. Buenos Aires: NOVEDUC, 2012.

JIMÉNEZ BECERRA, A.; FIGUEROA, H. Historia de la Universidad Pedagógica Nacional. Centro de Investigaciones de la Universidad Pedagógica, Universidad Pedagógica Nacional, Bogotá, 2002.

JIMÉNEZ EGUIZÁBAL et al. La política educativa y la naturaleza compleja de la educación. Nuevos enfoques epistemológicos, Revista española de pedagogía, Madrid, v. 64, n. 234, mayo-agosto, p. 249-272, 2006.

JONES, C. O. An Introduction to the Study of Public Policy. Belmont: Wadsworth. 1970.

KRIESI, H. Les démocraties occidentales. Una aproche comparée. Paris: Economica. 1994. 
LAITIN, D. ¿A dónde va la ciencia política? Reflexiones sobre la afirmación del profesor Sartori de que 'la ciencia política estadounidense no va a ninguna parte', Política y Gobierno, Ciudad de México, v. XI, n. 2, p. 361- 367, 2004.

LANDER, E. (Comp.). La colonialidad del saber, eurocentrismo y ciencias sociales. Buenos Aires: CLACSO, 2000.

LASSWELL, H. "The Policy Orientation." In: LERNER, D; LASSWELL, H. The policy sciences: recent developments in scope and method. Stanford: Stanford University Press, 1951.

LINDBLOM, E. The Science of Muddling Through, Public Administration Review, v. 19, n. 2, p. 79-88, 1959.

LUCCA, J. Debates y embates de la politología, Iconos, Revista de Ciencia Sociales, Quito, Ecuador, FLACSO, n. 30, p. 13-26, 2008.

MAINARDES, J. Reflexiones sobre el objeto de estudio de la política educacional. In: TELLO, C. (Org.). Los objetos de estudio en política educativa. Hacia una caracterización del campo teórico. Buenos Aires: Editorial Autores Argentinos, 2015.

MARÍN MARÍN, Á. Historia de la pedagogía en México, Correo del Maestro, Ciudad de México, v. 1, n. 11, p. 22-40, abril, 1997.

MIÑANA BLASCO, C. Producción y circulación el conocimiento en el campo de la política educativa y la gestión en América Latina. Universidad Nacional de Colombia, 2003. Inédito.

MCLUHAN, M. Understanding media: The extensions of man. Nueva York: McGrawHill, 1965.

MULLER, P. Les politiques publiques. Paris: PUF, 1990.

MULLER, P. L'analyse cognitive des politiques publiques: vers une sociologie politique de l'action publique. Revue Française de Science Politique, Paris, v. 50, n. 2, p. 189-208, 2000. 
MULLER, P.; SUREL, Y. L'analyse des politiques publiques. Paris: Editions Montchrestien, 1998.

NAZIF, M.; ROJAS FIGUEROA, A. La investigación educativa latinoamericana en los últimos diez años, Revista de Educación, Madrid, p. 21-41, Julio, 1997.

NEGRETTO, G. Nota del editor. El rumbo de la ciencia política, Revista Política y Gobierno, México, CIDE v. 9, n. 2, p. 347- 348, 2004.

NOHLEN, D. ¿Cómo enseñar ciencia política? Revista Republicana: política y sociedad, Guadalajara, v. 2, n. 2, p. 3-7, 2003.

NÚNEZ, I. La formación de docentes. Notas históricas. In: AVALOS, B. Profesores para Chile: Historia de un Proyecto. Santiago: Ministerio de Educación, 2002.

OXHRON, P. El arte de la ciencia política, Metapolítica, n. 49, septiembre-octubre, 2006.

PAVIGLIANITTI, N. Aproximaciones al desarrollo histórico de la política educacional, Revista Praxis educativa, Año II, n. 2, diciembre, p. 3-8, 1996.

PONTÓN RAMOS, C. Constitución conceptual de la educación como objeto de estudio y su impacto en la formación de profesionales de la educación, Perfiles Educativos, Mexico, Año XXIV, n. 97-98, p. 117-126, 2002.

PUIGGRÓS, A. Democracia y autoritarismo en la pedagogía Argentina y latinoamericana. Buenos Aires: Galerna, 1986.

RAMA, G. Educación, participación y estilos de desarrollo en América Latina. Buenos Aires: Kapelusz - CEPAL, 1984.

RESTREPO, B. Las Facultades de educación: evolución, situación actual y tendencias hacia el futuro, Revista Educación y pedagogía, Colombia, n. 10-11, I Semestre, p. 298-317, 1983.

ROIZ, J. Ciencia política hoy. Barcelona: Editorial Teide, 1982.

SABATIER, P. A.; JENKINS-SMITH, H. Special Issue: Policy Change and PolicyOriented Learing: Exploring an Advocacy Coalition Framework, Policy Sciences v. 21, p. 123-278, 1988. 
SABATIER, P. A.; JENKINS-SMITH, H. The Advocacy coalition framework: an assessment. In: SABATIER, P. A. (Ed). Theories of the policy process. Boulder: Westview Press, p. 117-166, 1999.

SANTOS, B. S. Renovar la teoría crítica y reinventar la emancipación social (encuentros en Buenos Aires). Buenos Aires: CLACSO, 2006.

SARTORI, G. ¿Hacia a dónde va la ciencia política?, Revista Política y Gobierno, México: CIDE, v. 9, n. 2, p. 349- 354., 2004.

SAVIANI, D. História das ideias pedagógicas no Brasil. Campinas: Autores Associados, 2007.

SOTOLONGO CODINA, P.; DELGADO DÍAZ, C. La revolución contemporánea del saber y la complejidad social. Buenos Aires: CLACSO, 2006.

SOUTHWELL, M. Psicología Experimental y Ciencias de la Educación. Notas de Historias y Fundaciones. La Plata: EDULP, 2003.

SUASNABAR, C. Pedagogos críticos, expertos en educación, tecno-políticos, o qué? A propósito de la relación entre intelectuales de la educación y política en los últimos 50 años. Ponencia presentada en el Seminario "Elites intelectuales y formación del Estado" realizado por el IDES, IDAES y UdeSA realizado del 28-30 de abril de 2009 en Buenos Aires.

TELLO, C. Las epistemologías de la política educativa como enfoque y la vigilancia y el posicionamiento epistemológico del investigador. Práxis Educativa, Ponta Grossa, v. 7, n. 1, p. 53-68, jan./jul. 2012.

TELLO, C. (Org.). Los objetos de estudio en política educativa. Hacia una caracterización del campo teórico. Buenos Aires: Editorial Autores Argentinos, 2014.

TELLO, C. El objeto de estudio en ciencias sociales: entre la pregunta y la hipótesis debates sobre las decisiones epistemológicas en un proyecto de investigación. Revista Cinta de Moebio, Faculdad de Ciencias Sociales Universidad de Chile, n. 42, p. 225-242, 2011. Disponible en: <http://www.revistas.uchile.cl/index. php/ CDM/article/viewArticle/18162/19020>. Acceso: 12 fev. 2012. 
TELLO, C.; MAINARDES, J. La posición epistemológica de los investigadores en Política Educativa: debates teóricos en torno a las perspectivas neomarxista, pluralista y pos-estructuralista. Archivos Analíticos de Políticas Educativas, v. 20, n. 9, p. 1 -33, 2012. Disponible en: 〈http://epaa.asu.edu/ojs/article/ view/988〉. Acceso: 24 mar. 2012.

TELLO, C.; MAINARDES, J. Pluralismos e investigación en política educativa: una perspectiva epistemológica. Revista Mexicana de Investigación Educativa, v. XX, n. 66, p. 763-788, 2015a.

TELLO, C.; MAINARDES, J. Revisitando o enfoque das epistemologias da política educacional. Práxis Educativa, v. 10, n. 1, pp. 153-178, 2015b.

TIRAMONTI, G. Después de los 90: agenda de cuestiones educativas, Cadernos de Pesquisa, Argentina- Flacso, 119, p. 71-83, julho, 2003.

UNESCO, International Conference on: Methods in Political Science, 13 September 1948-16 September 1948. Statement issued by the members of the Conference, 16 September 1948. Paris, UNESCO, 28 April 1949.

VERGARA ESTEVEZ, J. Las ciencias sociales latinoamericanas: desarrollo, crisis y perspectivas. SEMINARIO INTERNACIONAL DE LA COMISIÓN DE EPISTEMOLOGÍA Y POLÍTICA DE CLACSO, Santiago de Chile, 1991.

VITAR, A. Políticas de educación. Razones de una pasión. Buenos Aires: Miño y Dávila, 2006.

WALLERSTEIN, I. Impensar las ciencias sociales, México: Siglo XXI, 1999.

WEBER, M. El Político y el científico. Madrid: Alianza editorial, 1991 [1918].

Recebido: 15/08/2016

Recibido: 08/15/2016

Aprovado: 10/09/2016

Aprobado: 09/10/2016 